Itinéraires Itinéraires

Littérature, textes, cultures

\title{
Différence des sexes et désordre amoureux dans la littérature romantique française
}

\section{Maxime Foerster}

\section{(2) OpenEdition}

1 Journals

\section{Édition électronique}

URL : http://journals.openedition.org/itineraires/2455

DOI : $10.4000 /$ itineraires.2455

ISSN : 2427-920X

Éditeur

Pléiade

\section{Référence électronique}

Maxime Foerster, «Différence des sexes et désordre amoureux dans la littérature romantique française », Itinéraires [En ligne], 2014-2 | 2015, mis en ligne le 13 juillet 2015, consulté le 02 octobre 2020. URL : http://journals.openedition.org/itineraires/2455 ; DOI : https://doi.org/10.4000/itineraires. 2455

Ce document a été généré automatiquement le 2 octobre 2020.

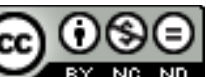

Itinéraires est mis à disposition selon les termes de la licence Creative Commons Attribution - Pas d'Utilisation Commerciale - Pas de Modification 4.0 International. 


\title{
Différence des sexes et désordre amoureux dans la littérature romantique française
}

\author{
Maxime Foerster
}

\section{Controverse sur la différence des sexes dans le romantisme français}

1 Concernant les questions relatives aux sexualités et aux identités de genre, il est étonnant de constater que les dernières études publiées sur le romantisme français développent deux perspectives en profonde contradiction. D'un côté, le recueil d'essais Masculinités en révolution de Rousseau à Balzac (Maira et Roulin 2013), ainsi que le livre Les Équivoques du genre: devenir homme et femme à l'âge romantique (Gutermann-Jacquet 2012), établissent une déconstruction des identités de genre et de la sexualité à l'œuvre dans les textes d'auteurs romantiques (Musset, Sand, Staël, Vigny), plaçant le romantisme français sous le signe d'un rapport critique et novateur vis-à-vis des valeurs patriarcales héritées de l'ancien régime, et d'un autre côté le Dictionnaire $d u$ romantisme (Vaillant 2012), insiste au contraire sur le rôle majeur du romantisme dans la préservation de l'éternel féminin et de la différence des sexes. Le dictionnaire ne contient pas une entrée sur les hommes, mais voici un extrait de celle sur les femmes:

En lisant tous les écrivains romantiques - romanciers, poètes, essayistes -, on constituerait sans peine une interminable anthologie qui ressasserait les mêmes lieux communs sur la femme: sa troublante proximité avec le divin, ses mystères insondables, les trésors que recèlent son âme et son cœur, la douce et envoûtante harmonie qui émane de sa voix et de tout son être. [...] Au regard de cette vulgate romantique sur la femme, très peu de voix discordantes se font entendre. (Vaillant 2012 : 250-251)

Le Dictionnaire du romantisme ne partage pas la déconstruction du féminin (encore moins du masculin, significativement absent du dictionnaire) engagée par les deux autres ouvrages contemporains, mais affirme au contraire que l'éternel féminin, en 
dépit de quelques voix discordantes et de la naissance du féminisme moderne, est plutôt fortifié que contesté par la majorité des auteurs romantiques. En somme, la question est posée par la discordance des publications contemporaines de savoir si la littérature romantique française est plutôt conservatrice, progressiste ou écartelée dans sa politique sexuelle.

3 En nous penchant sur les rapports de couples entre hommes et femmes dans la littérature romantique française, nous aimerions avancer qu'une des originalités du romantisme français est d'avoir profondément ébranlé la différence des sexes à travers l'illustration d'un désordre amoureux qui résulte de la tentative de s'opposer aux normes culturelles et sexuelles de la société française postrévolutionnaire.

\section{Le romantisme français comme espace de résistance et d'expérimentation}

Commençons par un aphorisme de Novalis pour introduire notre sujet, cité par George Gusdorf dans Du néant à Dieu dans le savoir romantique : "Chaos et Eros, voilà la meilleure définition du romantisme» (1983: 74). Bien avant que Rimbaud n'invite ses contemporains à être résolument modernes en réinventant l'amour, les romantiques avaient déjà lancé ce grand chantier de la modernité en s'attachant à redéfinir l'amour au moyen d'une nouvelle approche du féminin, du masculin et de la sexualité. Il ne s'agit pas de comprendre éros et chaos isolément, mais au contraire dans la dialectique qui les unit et dont il résulte, au niveau de la littérature romantique française, l'impression d'un grand désordre amoureux. Ce que traduit d'abord ce désordre amoureux, c'est d'une part la prolifération des hommes mélancoliques, des femmes d'esprit et des personnages androgynes dans la littérature romantique française, et d'autre part la récurrence des couples hétérosexuels qui ne se marient pas, ne font pas d'enfants et se rendent profondément malheureux. Lorsque Blanchot se penche sur le romantisme dans L'Entretien infini, il s'inspire également de Novalis pour théoriser un je-ne-sais-quoi du caractère romantique, à la fois fou et flou, et qui frappe avant tout par sa bizarrerie :

Il en résultera [...] ce caractère dit romantique qui, du reste, est très attrayant, dans la mesure où il lui manque précisément tout caractère, s'il n'est rien d'autre que l'impossibilité d'être quoi que ce soit de déterminé, de fixe, de sûr - d'où la frivolité, la gaieté, la pétulance, la folie : finalement, la bizarrerie et tout ce que Novalis condamnera, lorsqu'il reprochera lucidement à l'âme romantique de se rendre trop faible par dispersion et d'être efféminée. (Blanchot 1969 : 525)

Le mot queer n'est certes pas présent dans le texte, et pourtant il désigne déjà dans l'Angleterre victorienne quelque chose de bizarre, d'étrange et de malsain, faisant écho aux expressions de manque de caractère, de folie, de faiblesse et d'efféminement qu'on trouve sous la plume de Blanchot. Il n'est question que de négativité dans l'écriture de Blanchot pour saisir ce caractère romantique qui se caractérise, précisément, par son manque de consistance et de cohérence ainsi qu'une efflorescence confinant à la superficie et à l'éphémère, à une identité fragmentaire, inachevée dans sa masculinité et jouant dangereusement avec la folie. Nous reviendrons en conclusion de cet essai sur l'éclairage que peut apporter la littérature romantique française au regard des questions contemporaines sur les identités de genre et les familles atypiques, mais nous devons à présent expliquer le fait d'avoir recours à deux autres termes anachroniques 
dans notre réflexion sur le romantisme: nous utiliserons en effet les termes de sexualité et d'hétérosexualité pour penser la façon dont la littérature romantique française a œuvré à une réinvention de l'amour, quand bien même les mots de « sexualité » (comme le rappelle Alain Corbin dans L'Harmonie des plaisirs : les manières de jouir du siècle des Lumières à l'avènement de la sexologie) et d' "hétérosexualité " (dont l'histoire a été ébauchée par Jonathan Ned Katz dans L'Invention de l'hétérosexualité) n'existaient pas dans la première moitié $d u x^{e}{ }^{e}$ siècle et devront attendre la propagation de la sexologie pour être théorisés et gagner en intelligibilité par-delà le cercle des médecins vers la fin du même siècle.

L'anachronisme est avéré, certes, mais c'est précisément parce que la littérature romantique française s'offre comme espace alternatif pour opposer un désordre amoureux à la politique de remise en ordre du patriarcat menée par l'Empire puis par la Restauration qu'il peut être perçu comme un mouvement culturel brouillant volontairement l'édifice de la différence des sexes et retardant autant que possible ce que Foucault avait analysé comme le grand quadrillage de l'individu livré à de nouvelles formes de surveillance via la pédagogie et la discipline. Comme l'a brillamment démontré Déborah Gutermann-Jacquet dans son livre Les Équivoques du genre: devenir homme et femme à l'âge romantique (2012), le romantisme est un texte d'opposition qui s'inscrit contre un contexte culturel et politique visant à solliciter le droit pour encadrer la nouvelle rhétorique et les nouveaux standards d'une masculinité et d'une féminité en manque de substance après la grande secousse des revendications portées lors de la Révolution française. Il s'agit de maintenir l'héritage traditionnel de la différence des sexes et de la domination masculine tout en le promouvant à travers les habits neufs de la modernité, par le nouveau langage des dispositifs d'alliance, de l'éducation des enfants et du droit civil. Un exemple concret de cette nouvelle rhétorique de promotion de la différence des sexes a été étudié par Élisabeth Badinter dans L'Amour en plus (2010): une perspective stable et conservatrice de l'identité féminine a été rattachée à la construction moderne, rousseauiste, du concept d'instinct maternel au XVIII et surtout au XIX ${ }^{e}$ siècle. C'est dans cette fabrique des hommes et des femmes que se joue la nouvelle norme hétérosexuelle et que le romantisme fait figure, en littérature mais aussi au niveau culturel, d'espace de résistance et d'identités sexuelles alternatives :

La fabrique des hommes et des femmes à l'époque romantique suppose ainsi un aller-retour entre les sources normatives, les sources intimes et les sources littéraires. D'une part parce que l'univers des prescriptions édictées par la littérature grise fournit des énoncés dont la force discursive induit la production d'un «standard» et d'un référent, tandis que l'univers romanesque prescrit un contre-modèle également investi. (Gutermann-Jacquet 2012 : 14)

7 Avant que la sexologie n'accouche d'une nouvelle terminologie, l'État français, qu'il se soit revendiqué de la Terreur, de l'Empire ou de la Monarchie, s'est retrouvé uni dans sa politique de réhabilitation de la famille patriarcale en renforçant la précarité juridique de la femme et sa dépendance vis-à-vis de l'homme par le biais de leur


pas encore nommées qu'elles n'en sont pas moins à l'œuvre, en gestation, dans la façon dont l'État dénie aux femmes l'accès au statut de citoyen à part entière : on assiste à la mise en place de l'hétérosexualité non pas comme sexualité procréatrice mais bel et bien comme régime politique, assignant à chaque être humain une essence identitaire fixe et un destin social balisé en fonction de son sexe de naissance. La naissance de 
l'hétérosexualité comme régime politique est le trait fondamental de la modernisation de la domination masculine: Thomas Laqueur, en analysant le passage du «one-sex model » à celui du «two-sex model » dans Making Sex (1990), fait du concept de la différence des sexes l'élément crucial de l'aliénation d'un sexe par l'autre, et rejoint la perspective de Monique Wittig pour qui, dans La Pensée straight ([1992] 2007), la création même de la catégorie de sexe sert de marqueur pour enfermer les femmes dans l'enclos de la différence des sexes.

8 La littérature romantique française peut être perçue comme une sorte d'atelier dédié à promouvoir des identités de genre, des sexualités, des couples amoureux qui n'illustrent pas l'évidence de la différence des sexes mais cherchent au contraire à s'en affranchir, à en brouiller la naturalité et la légitimité. Si nous avons choisi d'utiliser l'expression de désordre amoureux, c'est pour sortir le chaos romantique, décrit plus haut par Gusdorf et Blanchot sous l'égide de Novalis, de sa négativité : si le désordre amoureux du romantisme français est sans destination idéologique établie, il n'en est pas moins guidé par la volonté d'inventer un ordre alternatif (d'où la notion de désordre au regard de ce qu'il essaie de subvertir) et d'expérimenter une affirmation de soi et du sentiment amoureux qui composent une ligne de fuite vis-à-vis de la différence des sexes et des contraintes politiques et sociales qui lui sont inhérentes.

Ce désordre amoureux se traduit par le rejet de tout ce qui confère la normalité et l'acceptation sociale, c'est-à-dire rejet de l'institution du mariage, du devoir de procréation, d'une virilité ostentatoire pour les hommes et d'une féminité naturelle pour les femmes. Dans la littérature romantique française, les exemples abondent d'hommes efféminés, mélancoliques, refusant une brillante carrière voulue par leur père (l'Adolphe de Constant et l'Octave de Musset rejettent le libertinage de la génération précédente et "ratent » leur vie en refusant la carrière voulue par leur père), de femmes d'esprit accusées d'être contre-nature (la Delphine de Staël, la Mathilde de Stendhal, la Lélia de Sand), de couples amoureux qui ne parviennent pas à valider les deux cases de la normalité : l'institution du mariage et la procréation du couple. J'aimerais articuler l'intelligibilité de ce désordre amoureux en développant la thèse suivante : la littérature romantique française est un site de résistance à la mise en place d'une norme sexuelle s'appuyant au début du XIX ${ }^{e}$ siècle sur une politique de promotion de la famille monogame, procréative et bourgeoise, relayée dans la seconde moitié $\mathrm{du} \mathrm{XIX}^{\mathrm{e}}$ siècle par l'influence du positivisme dont les versants médicaux, psychiatriques et sexologiques tendent à définir et soigner toutes les pathologies sexuelles dans le but d'édifier une hétérosexualité saine au service d'une nation virile et régénérée ${ }^{2}$.

10 Ainsi conçu, le désordre amoureux à l'œuvre dans le romantisme n'est simplement pas le culte de la pulsion suicidaire ni la passion poussée jusqu'à la destruction mais plutôt la constitution d'un espace littéraire comme lieu de résistance : ce désordre traduit le projet de réinventer l'amour suite au rejet de l'hétérosexualité définie comme un contrat social assignant aux citoyens, selon leur sexe de naissance, une identité de genre et une sexualité définies par des « lois de la nature » nulle part inscrites dans la nature mais prescrites par des penseurs en son nom. La spécificité du romantisme français, au regard d'autres romantismes européens, est d'avoir été plus radical dans sa contestation de la monarchie, de l'Église et du capitalisme. Dans les Fondements du savoir romantique, Gusdorf, commentant la trajectoire politique de Victor Hugo, conclut en écrivant que «le romantisme, en France, fera cause commune avec l'idéologie de 
gauche, alors qu'en Allemagne il se fige dans une attitude traditionaliste et catholicisante " (Gusdorf 1982 : 152). Le poète lui-même avait posé ce diagnostic, qui engageait bien plus que lui-même: "Je mis un bonnet rouge au vieux dictionnaire ${ }^{3}$." Ce radicalisme propre au romantisme français se traduit aussi, par-delà une critique de l'oppression politique, de l'injustice sociale et du fanatisme religieux, par le projet d'une réinvention de l'amour pour s'opposer à l'héritage contre-révolutionnaire de l'Empire et de la Restauration qui sont revenus sur le droit au divorce et ont entériné la place des femmes dans la sphère domestique, au service de la famille devenue une mise en abyme de la nation française (en 1804, le Code civil accule la femme à l'incapacité juridique sous l'Empire et en 1816 la Restauration interdit le droit de divorcer par la loi Bonald $)^{4}$.

\section{Une hétérosexualité globalement dysfonctionnelle}

11 De quel romantisme français parlons-nous quand nous défendons l'idée que la littérature romantique française s'est efforcée de résister à la mise en place de l'hétérosexualité comme régime politique en opposant l'expérience d'un désordre amoureux généralisé ? Quand bien même nous ne citerons dans cet article que Senancour, Stendhal, Staël, Constant, Sand et Gautier, nous aurions pu tout aussi bien défendre la thèse d'une hétérosexualité dysfonctionnelle présente dans les textes de Musset et Vigny, de Mérimée et Hugo, y compris dans les écrits des romantiques se revendiquant du catholicisme et de la monarchie, comme Chateaubriand, par exemple, dont le roman René (1802) a mis en pleine lumière une hétérosexualité pour le moins problématique à travers l'amour incestueux entre une sœur et un frère dont la masculinité est particulièrement châtrée. Ainsi, peu importe l'antiféminisme ou l'antirépublicanisme de certains auteurs romantiques, ce n'est pas dans une interprétation psycho-biographique des écrivains qu'on trouvera le désordre amoureux, mais bel et bien dans leurs œuvres avec un constat doublé d'une question : pourquoi est-ce que les couples hétérosexuels qui fournissent si souvent l'intrigue de la littérature romantique française, ne se marient pas, sont d'une masculinité et/ou d'une féminité problématiques, ne font pas d'enfants et sont en proie à l'opprobre ou à la marginalité sociale? C'est de la récurrence de ce dysfonctionnement hétérosexuel dans l'ensemble de la littérature romantique française que nous pensons rendre compte grâce au concept du trouble hétérosexuel ${ }^{5}$.

Le trouble hétérosexuel vient du fait que ce sont les hétérosexuels, souvent en couples, qui sont mis en avant dans la littérature romantique française pour illustrer la tentative de réinventer l'amour en s'opposant à l'opinion commune, à l'institution du mariage et au devoir de l'enfantement dans le cadre d'une famille monogame et patriarcale. Ces personnages hétérosexuels, en cherchant à vivre un amour hors norme, finissent par faire de leur hétérosexualité ce que Foucault, dans Les mots et les choses, catégorisait comme une hétérotopie :

Les utopies consolent: c'est que si elles n'ont pas de lieu réel, elles s'épanouissent pourtant dans un espace merveilleux et lisse; elles ouvrent des cités aux vastes avenues, des jardins bien plantés, des pays faciles, même si leur accès est chimérique. Les hétérotopies inquiètent, sans doute parce qu'elles minent secrètement le langage, parce qu'elles empêchent de nommer ceci et cela, parce qu'elles brisent les noms communs ou les enchevêtrent, parce qu'elles ruinent d'avance la "syntaxe », et pas seulement celle qui construit les phrases, - celle 
moins manifeste qui fait « tenir ensemble » (à côté et en face les uns des autres) les

mots et les choses. (Foucault $1996: 9$ )

L'hétérosexualité hors norme des antihéros romantiques partage avec l'hétérotopie le fait de produire de l'inquiétude puisqu'elle provoque le désordre en résistant aux identités et institutions sociales, et d'introduire de la confusion à la fois au niveau du langage et de l'ordre symbolique en troublant volontairement la syntaxe du masculin et du féminin. Par exemple, quand un homme en proie aux crises de nerfs, comme Léonce, aime et est aimé d'une femme d'esprit, comme Delphine, dans le roman éponyme de Mme de Staël, il en résulte une torsion du langage rendant perméable et relatif ce qui relève du masculin et du féminin. Lorsque Léonce écrit à Delphine : «Je te jure par tout ce qu'il y a de plus sacré sur la terre, de te respecter comme un frère " (Staël [1802] 2000, vol. $2: 12$ ), il demeure une ambiguïté sur ce à quoi le mot frère fait référence : rien ne permet de déterminer s'il respectera Delphine comme si elle était son frère, ou comme si c'était lui qui était le sien. Deux pages plus tard, même exemple d'androgynie textuelle pour décrire la façon dont Léonce prend soin de Delphine: «Depuis que Delphine est presque convalescente, il invente mille soins nouveaux comme l'amie la plus attentive» (Staël [1802] 2000 : 14). Dans ce cas, Léonce, tout homme qu'il est, est associé au statut féminin de "l'amie la plus attentive" pour signifier une nouvelle confusion dans la dialectique du féminin et du masculin à l'œuvre dans ce couple hétérosexuel.

Dans la littérature romantique française, l'androgynie textuelle témoigne de l'inscription stylistique d'une confusion des genres troublant l'évidence naturelle de la différence des sexes. L'androgynie textuelle peut commencer à partir du changement de sexe par pseudonymes : Aurore Dupin devient George Sand, Jules Barbey D'Aurevilly devient Maximilienne de Syrène et, dans une nouvelle de Baudelaire, Samuel Cramer signe ses poèmes du nom de Manuela de Monteverde. Le changement de sexe le plus connu est celui de Théodore de Sérannes, c'est-à-dire Madeleine de Maupin qui, au gré de son identité de genre fluctuante, varie les accords de sa conjugaison : le masculin de «je me croirais manqué » et «tant que je ne serai pas parvenu à mon but » (Gautier [1835] 1999: 68) contraste avec le féminin de « si j'étais restée chez moi » (Ibid.: 344) et "je restai confondue" (Ibid.: 351). Quand bien même Madeleine de Maupin est un exemple d'ambiguïté sexuelle poussé à son extrême, ce sont plusieurs personnages romantiques qui peuvent se reconnaître dans sa confession: "Je perdais insensiblement l'idée de mon sexe» (Ibid.: 356). C'est la perte de l'idée d'un sexe assigné qui se traduit dans le style par une androgynie textuelle, et c'est l'envie d'expérimenter une existence romanesque qui inspire aux personnages romantiques le projet de survoler les conventions sociales et sexuelles. Si Madeleine devient Théodore, c'est, comme elle l'explique elle-même, parce qu'elle vit dans une société qui promet aux femmes l'ennui, la soumission et la mièvrerie d'une existence domestique.

\section{Mélange des genres (littéraires et sexuels)}

Dans son analyse de Lélia, Naomi Schor pose la question suivante: "Is the scandal of Lélia a scandal of genre rather than of gender?" (Schor 1993: 56). Pour Schor, la «faute » qu'on reprochera à Sand est de ne pas s'être tenu à l'idéalisme de l'allégorie pour s'abîmer de temps en temps dans le réalisme, d'où le mélange des genres typique de son œuvre et d'autres œuvres romantiques. Pour nous, la coïncidence entre le 
mélange des genres littéraires et le mélange des identités de genre (qui fait l'homme, qui fait la femme dans le couple hétérosexuel romantique ?) témoigne du fait que ce qui fait problème dans les couples hétérosexuels, c'est l'impossibilité de vouloir faire le deuil d'un amour à inventer au nom du principe du réel, d'où le va-et-vient entre idéalisme et réalisme qui ne font pas meilleur ménage que l'homme et la femme en amour. C'est que précisément, dans la littérature romantique française, l'androgynie textuelle associe la confusion de l'identité de genre (masculin/féminin) à la confusion de genre littéraire : ces deux confusions ne se retrouvent pas liées par coïncidence mais se confortent en déclinant le désordre au niveau du genre et du gender.

De même que l'idée de sexe se perd sensiblement à la fois par le thème et le style de l'amour romantique, ce flou sexuel trouve écho dans un autre chaos, volontairement orchestré lui aussi, par un texte qui emprunte aux divers genres littéraires pour composer, en toute indépendance vis-à-vis des règles du classicisme, une littérature résolument transgenre. L'instabilité de l'identité de genre est soutenue, et comme amplifiée, par l'impossibilité d'ancrer le texte romantique dans un genre littéraire clairement défini. Ainsi Corinne ou l'Italie, de Mme de Staël (1802), n'est pas seulement un roman puisqu'il incorpore des pages de correspondance, des passages dignes d'un guide touristique de l'Italie et des œuvres poétiques de l'artiste Corinne ${ }^{6}$. De même pour Isidora, roman de Sand moins connu que Lélia mais pas moins atypique qui intègre dans sa prose romanesque les essais de Jacques, un chercheur en sciences humaines se proposant de méditer sur la question de la différence des sexes : « La femme est-elle ou n'est-elle pas l'égale de l'homme dans les desseins, dans la pensée de Dieu ? La question est mal posée ainsi, il faudrait dire : l'espèce humaine est-elle composée de deux êtres différents, l'homme et la femme?" (Sand [1846] 1894: 8). À l'époque où elle publie Isidora (1846), Sand, désillusionnée par les utopistes et les penseurs sociaux (Leroux, Lamennais, Saint-Simon) avec qui elle avait cru pouvoir rêver d'un monde meilleur, crée le personnage de Jacques pour se moquer de l'aspect trop abstrait, trop théorique de ses recherches sur la question de la différence des sexes, démontrant que ses bonnes intentions ne suffisent pas à l'éclairer tant qu'il ne s'attachera pas personnellement, de manière intime, à la question du masculin et du féminin.

\section{Une intelligibilité différente du désordre amoureux selon le sexe de l'auteur romantique?}

17 À ce stade de notre réflexion, il nous faut anticiper une remarque probable de notre lecteur qui fera l'hypothèse que notre hétérosexualité dysfonctionnelle est facilement soluble, voire réductible, à une approche féministe se basant sur l'interprétation d'une sélection de textes pour vouloir associer le romantisme français à la dynamique d'émancipation des femmes qui a ponctué le XIX siècle. La sensibilité féministe est indéniablement une composante de cette résistance à une hétérosexualité normative, mais la littérature romantique française, dans son effort pour réinventer l'amour, et tout en ayant conscience de l'injustice de l'exploitation de la femme par l'homme, ne souhaite pas se limiter à libérer les femmes de la domination masculine mais vise plus globalement à réinventer l'amour par-delà la matrice de la différence des sexes, c'est-àdire en perdant le sexe anatomique comme marqueur identitaire du sujet. En ce sens, les hommes hétérosexuels comme Léonce ou Sténio, par exemple, tout en étant des oppresseurs du simple fait de leur sexe, n'en sont pas moins décrits comme victimes, 
d'une part, du devoir d'être viril alors que leur masculinité est problématique, et d'autre part, d'aimer une femme que la société juge contre-nature en raison de ses prétentions intellectuelles ou de ses activités artistiques. Ainsi, le statut privilégié d'homme ne dispense pas les personnages masculins de souffrir à la fois contre et avec leurs amantes de l'extrême difficulté pour leur couple hétérosexuel de s'aimer en dehors des normes hétérosexuelles mises en place par la société en voie de modernisation. On citera ici deux passages de Delphine et de Lélia pour mettre en évidence que le trouble hétérosexuel ne se limite pas à la libération de la femme, mais inclut aussi l'affranchissement des hommes au devoir (au fardeau) d'être exemplairement viril. Ainsi Delphine constate qu'elle forme un couple étrange avec Léonce :

Ce bonheur ou ce calme dont je jouis, que deviendraient-ils néanmoins, si par un renversement bizarre c'était moi, faible femme, moi dont la destinée réclame un soutien, qui saurais mépriser l'opinion des hommes, tandis que l'être fort, celui qui doit me guider, celui qui doit me servir d'appui, aurait horreur du moindre blâme? (Staël [1802] 2000, vol. $1: 127$ )

L'usage du conditionnel est pur euphémisme de la part de Delphine, puisque le roman décrit très exactement les conséquences dramatiques de cette inversion des genres dans le couple hétérosexuel.

Quant à Sténio, il se déclare prêt lui aussi à vivre une inversion des rôles dans le couple qu'il souhaite former avec Lélia :

Mais moi, je n'ai pas la volonté qui fait la grandeur et l'énergie du rôle viril. [...] Eh bien! Lélia, c'est pour cela que je vous aime, vous avez pris mon rôle, que les hommes vous refusaient. Loin de répudier le vôtre, je vous le demande à genoux. (Sand [1833] $2003:$ 225)

Puisque la réinvention de l'amour dans le romantisme français passe par une confusion volontaire des sexes entre eux, la résistance à l'hétérosexualité comme régime politique est un enjeu non seulement pour les hommes et les femmes hétérosexuels, mais aussi pour tous les autres personnages romantiques que leur marginalité sexuelle place hors de l'hétérosexualité: l'hermaphrodite Fragoletta, l'ange androgyne Séraphita, l'impuissant Octave de Malivert, la bisexuelle transgenre Madeleine de Maupin, l'inverti Claude Gueux, etc. La réinvention de l'amour dans la littérature romantique française résiste autant que possible à la vaste entreprise de sexage des individus pour les ancrer dans leur différence sexuelle, politique et identitaire ${ }^{7}$.

21 Il faut se garder de croire à un courant féministe qui, dans le romantisme français, serait essentiellement le domaine des femmes écrivains et qui délimiterait par conséquent la portée du trouble hétérosexuel ${ }^{8}$. La résistance à un modèle hétérosexuel normatif traverse l'ensemble de la littérature romantique française, incorpore - même indirectement - tout en le dépassant l'analyse féministe de la domination masculine et se retrouve articulée dans les œuvres des auteurs des deux sexes. Certes, cela ne veut aucunement dire que toutes les œuvres écrites par tous les auteurs romantiques français sont engagées dans une critique radicale de l'hétérosexualité décriée comme contrat social, mais cette critique est assez largement portée pour ne pas être réduite à un courant féministe du romantisme français quand elle en est une spécificité. Le contraste entre une citation de Benjamin Constant et une autre de Senancour nous permettra d'articuler précisément d'une part la diversité de sensibilité vis-à-vis de la réinvention de l'amour à l'intérieur du romantisme français, et d'autre part que cette diversité confirme plus qu'elle n'infirme le fait que la réinvention de l'amour comme 
désordre amoureux est au cœur de la littérature romantique française. Dans sa « Lettre sur Julie ", voici ce que Constant exprime au sujet de l'amour et des femmes :

Presque toutes les femmes parlent bien sur l'amour : c'est la grande affaire de leur vie; elles y appliquent tout leur esprit d'analyse, et cette finesse d'aperçu dont la nature les a douées pour les dédommager de la force. Mais comme elles ont un intérêt immédiat, elles ne sauraient être impartiales. Plus elles ont de pureté d'âme, plus elles sont portées à mettre aux liaisons de ce genre une importance, je ne dirai pas, pour ne scandaliser personne, exagérée, mais cependant en contraste avec l'état nécessaire de la société. (Constant [1829] 1957 : 91-92)

Dans une formule de prétérition qui ne manque pas d'ironie, Constant reproche aux femmes de porter à l'amour une attention si vive qu'elle finit par menacer l'ordre social. Établissant que l'amour est la grande affaire des femmes, Constant fait l'économie d'un questionnement qui pourrait l'amener à réfléchir sur les causes qui font que les femmes sont amenées à être exclusivement occupées d'amour. Autre question qu'il ne soulève pas, lui qui accuse les femmes d'être parties prenantes: qu'est-ce que l'impartialité en amour? La conclusion tirée par Constant ressemble à une mise en garde : à trop se mêler d'amour, qui plus est de façon partiale, les femmes portent atteinte à "l'état nécessaire de la société ». Où l'on revient à la définition du romantisme comme désordre amoureux doublé d'une contestation sociale, c'est-à-dire une tentative de réinventer l'amour qui ne peut qu'ébranler l'ordre social. Tandis que Constant impute aux femmes le risque de désordre social et amoureux, la littérature romantique française montre que les hommes aussi partagent avec les femmes une vive attention à l'amour et à ses conséquences sociales. Chateaubriand, dans ses Mémoires, avait loué Lélia tout en reprochant à Sand d'écrire une littérature propice au désordre, mais l'amour incestueux unissant René à sa sœur n'était-il pas moins propice au même genre de désordre? De même, Constant n'a-t-il pas lui aussi, dans Adolphe (1816), déstabilisé l'état nécessaire à la société en décrivant l'amour d'une femme plus riche et plus âgée pour un garçon de vingt-deux ans?

L'extrait suivant, tiré d'Obermann, dévoile une autre approche de la question des femmes et de l'amour:

Femmes aimables, ne sentirez-vous pas tout ce que vous valez? - Le besoin d'aimer ! - Il ne vous excuse pas. Le premier des besoins est de ne pas s'avilir, et les besoins du cœur doivent eux-mêmes vous rendre indifférent quiconque n'a de l'homme autre chose que de ne pas être femme. [...] Si l'homme est l'ami naturel des femmes, les femmes n'ont souvent pas de plus funeste ennemi. Tous les hommes ont le sens de leur sexe ; mais attendez celui qui en a l'âme. (Senancour [1804] 1984 : 404)

Ce qui mène au trouble hétérosexuel dans ce passage, c'est qu'un narrateur masculin conseille aux femmes de ne pas aimer un homme qui ne saurait définir sa masculinité autrement que comme le fait de ne pas être femme. Senancour reprend avec Constant le thème des femmes dévouées à l'amour mais il les exhorte à ne pas aimer un homme si l'amour est avilissant pour elle, ce qui est immanquablement le cas si l'homme se définit en tant qu'homme plus par son sexe (négativement : ne pas être femme) que par son âme (qu'il a en partage avec les femmes). Ce faisant, le narrateur suggère que les femmes devraient ne s'autoriser à aimer un homme que s'il est capable de se définir autrement que comme étant l'opposé de la femme, précisément quand il peut la rejoindre par son âme au lieu de la circonscrire par son sexe. C'est tout l'enjeu de la réinvention de l'amour que de redéfinir la dialectique du féminin et du masculin pour que le couple hétérosexuel ne résulte plus de la complémentarité de deux sexes 
radicalement différents mais repose au contraire sur une "symétrie éclairée », pour reprendre une expression de Mme de Staël. Les perspectives de Constant et Senancour divergent sur la question de l'amour et des femmes, Constant liant les femmes à l'amour en leur demandant de ne pas trop exagérer sous peine de troubler l'ordre social tandis que Senancour ouvre la question de l'amour aux femmes et aux hommes en souhaitant un bouleversement de la dialectique du féminin et du masculin sans s'alarmer de ses possibles conséquences sociales. Les deux auteurs, néanmoins, ont conscience que le romantisme est l'espace littéraire d'une controverse des sexes et peut-être de sa résolution.

En comparant deux citations, une de Mme de Staël, tirée de la seconde préface aux Lettres sur Rousseau, et une de Stendhal, tirée du chapitre sur l'éducation des femmes dans son essai De l'amour, nous verrons clairement une complicité dans leurs perspectives sur ce qui est problématique dans l'amour hétérosexuel et comment ce problème peut être résolu. Mme de Staël plaide ainsi pour que les hommes cessent d'avoir peur d'aimer une femme aussi intelligente qu'eux :

Que devrait-on penser d'un époux assez orgueilleusement modeste pour aimer mieux rencontrer dans sa femme une obéissance aveugle qu'une symétrie éclairée? Les plus touchants exemples de l'amour conjugal ont été donnés par des femmes dignes de comprendre leurs maris et de partager leur sort, et le mariage n'est dans toute sa beauté que lorsqu'il peut être fondé sur une admiration réciproque. Néanmoins beaucoup d'hommes préfèrent les femmes uniquement consacrées aux soins de leur ménage ; et pour plus de sûreté à cet égard, ils ne seraient pas fâchés qu'elles fussent incapables de comprendre autre chose. (Staël [1788] $2008: 40-41$ )

À quoi Stendhal répond, sans citer Staël mais animé du même raisonnement et du même souci de prolonger l'intensité et la durabilité des sentiments :

Quel est l'homme dans l'amour ou dans le mariage qui a le bonheur de pouvoir communiquer ses pensées telles qu'elles se présentent à lui, à la femme avec laquelle il passe sa vie ? Il trouve un bon cœur qui partage ses peines, mais toujours il est obligé de mettre ses pensées en petite monnaie s'il veut être entendu, et il serait ridicule d'attendre des conseils raisonnables d'un esprit qui a besoin d'un tel régime pour saisir les objets. La femme la plus parfaite, suivant les idées de l'éducation actuelle, laisse son partenaire isolé dans les dangers de la vie et bientôt court le risque de l'ennuyer. Quel excellent conseiller un homme ne trouverait-il pas dans sa femme si elle savait penser ! (Stendhal [1822] 1959 : 214-215)

Staël et Stendhal, en fins analystes de l'amour et des enjeux de sa réinvention, se trouvent en harmonie lorsqu'ils estiment que les hommes ont autant à gagner que les femmes qu'ils aiment à les traiter en égales, à désirer qu'elles soient aussi libres qu'eux de cultiver leur intelligence et de s'épanouir loin des contraintes assignées au deuxième sexe. La similarité de leurs thèses prouve qu'il n'est pas pertinent de faire jouer un romantisme féministe et minoritaire, celui des "bas-bleus", contre le romantisme majoritaire et masculin, qui serait indifférent, voire opposé à la réinvention de l'amour et à la composante féministe qui lui est inhérente.

Dans ses Lettres sur Rousseau, Mme de Staël, pourtant grande admiratrice de l'auteur du Contrat Social, se permet de critiquer ce dernier quant à sa position sur les femmes et rejoint Senancour sur l'intérêt de définir quelqu'un par son âme au lieu de son sexe :

Le seul tort qu'au nom des femmes je reprocherais à Rousseau, c'est d'avoir avancé, dans une note de sa Lettre sur les spectacles, qu'elles ne sont jamais capables de peindre la passion avec chaleur et vérité. Qu'il leur refuse, s'il le veut, ces vains talents littéraires, qui, loin de les faire aimer des hommes, les mettent en lutte avec eux; qu'il leur refuse cette puissante force de tête, cette profonde faculté 
d'attention dont les grands génies sont doués : leurs faibles organes s'y opposent, et leur cœur, trop souvent occupé, s'empare sans cesse de leur pensée, et ne la laisse pas se fixer sur des méditations étrangères à leur idée dominante ; mais qu'il ne les accuse pas de ne pouvoir écrire que froidement, de ne savoir pas même peindre l'amour. C'est par l'âme, l'âme seule, qu'elles sont distinguées. (Staël [1788] 2008 :

49)

Ce n'est pas sans ironie que Mme de Staël se présente comme étant d'accord avec Rousseau pour dire que les femmes, à cause de la faiblesse de leurs organes, ne peuvent rivaliser avec les hommes dans le domaine intellectuel alors même qu'elle s'exprime dans un essai philosophique consacré au grand écrivain suisse. En maintenant contre Rousseau que les femmes écrivains savent décrire la passion avec chaleur et vérité, elle va plus loin encore en suggérant que ces dernières, chez qui le cœur s'empare toujours de la pensée, se retrouvent, précisément pour cette raison, à l'avant-garde du romantisme par cet entremêlement de la raison du cœur et de celle de l'âme. Mais c'est surtout la dernière phrase qui démontre le radicalisme du romantisme français dans sa réinvention de l'amour: ce n'est pas par le sexe, mais par son âme, qu'il faut appréhender l'individu.

\section{L'écriture romantique de ce « sexe à part qui n'a pas encore de nom »}

L'exaltation de l'âme contre une assignation identitaire à son sexe anatomique ne traduit en rien une perspective platonicienne qui ferait du corps le tombeau de l'âme et inviterait le sujet romantique à se dépouiller du poids du corps via le rejet du sexe. Le sexe anatomique n'est pas la métonymie du corps et ne renvoie pas à l'énergie érotique ; il est au contraire conçu comme ce qui, paradoxalement, «castre » le corps du sujet romantique en le fixant dans une identité déterminée à l'essence immuable quand ce dernier n'aspire qu'à être le récipient et le catalyseur d'énergies vitales le traversant et le faisant communier avec ce qui le dépasse.

Lorsque Senancour et Mme de Staël appellent à réhabiliter l'âme contre le sexe, il ne s'agit pas de promouvoir l'ascétisme mais au contraire de libérer le corps du sexage qui entrave sa capacité à accueillir et ressentir un souffle propre à le faire sortir de sa finitude. C'est peut-être Madeleine de Maupin qui résume le mieux ce rejet de la différence des sexes non pas pour mépriser le corps mais pour embrasser au contraire la (con)fusion du corps et de l'âme: "À force de vouloir nous empêcher d'être romanesques, l'on nous rend idiotes. Le temps de notre éducation se passe non pas à nous apprendre quelque chose, mais à nous empêcher d'apprendre » (Gautier [1835] 1999 : 222) et «Je suis d'un troisième sexe à part qui n'a pas encore de nom : au-dessus ou au-dessous, plus défectueux ou supérieur : j'ai le corps et l'âme d'une femme, l'esprit et la force de l'homme, et j'ai trop ou pas assez de l'un et de l'autre pour me pouvoir accoupler avec l'un d'eux" (Ibid.: 356). En refusant de se définir par son sexe anatomique de femme, Madeleine de Maupin / Théodore de Sérannes s'affranchit de la syntaxe de la différence des sexes, ce qui lui permet de braver l'interdiction d'être romanesque et de résister à l'injonction de l'union hétérosexuelle avec un sujet qui serait clairement sexué.

32 Faisant écho à la définition foucaldienne de l'hétérotopie et anticipant déjà l'amoralisme de Nietzsche, Lélia donne une perspective globale à son statut infamant 
de femme frigide et satanique en répondant à Sténio qui la soupçonne d'être mécréante :

Vous demandez si j'adore l'esprit du mal. L'esprit du mal et l'esprit du bien, c'est un seul esprit, c'est Dieu ; c'est la volonté inconnue et mystérieuse qui est au-dessus de nos volontés. Le bien et le mal, ce sont des distinctions que nous avons créées. (Sand [1833] $2004:$ :15) femmes du romantisme français, qu'on ne peut pas présenter le romantisme comme une force réactionnaire qui s'oppose à la Révolution française pour rappeler le poids de l'histoire et le respect des traditions. Au contraire, en se consacrant à la réinvention de l'amour, le romantisme français a relevé le défi de la modernité en proposant une nouvelle dialectique du féminin, du masculin et de la sexualité, dialectique qui a été 
perçue comme chaotique mais dont le désordre résulte d'une résistance moderne au renouvellement de l'ordre patriarcal.

\section{BIBLIOGRAPHIE}

Badinter, Élisabeth, 2010, L'Amour en plus. Histoire de l'amour maternel (XVII $-\mathrm{XX} \mathrm{X}^{e}$ siècle), Paris, Flammarion.

Baudelaire, Charles, 2004, Euvres complètes, Paris, Robert Laffont.

Blanchot, Maurice, 1969, L'Entretien infini, Paris, Gallimard.

Causse, Michèle, 2000, Contre le sexage, Paris, Balland.

Chateaubriand, [1802] 1992, René, Paris, Gallimard.

Constant, Benjamin, 1957, Le Romantisme, Paris, Edito.

-, [1816] 2005, Adolphe, Paris, Gallimard.

Corbin, Alain, 2010, L'Harmonie des plaisirs : les manières de jouir du siècle des Lumières à l'avènement de la sexologie, Paris, Perrin.

Delon, Michel, et Melonio Françoise (dir.), 2000, Mme de Staël : Actes du colloque de la Sorbonne du 20 novembre 1999, Paris, Presses de l'Université Paris-Sorbonne.

Foucault, Michel, [1966] 1996, Les Mots et les Choses, Paris, Gallimard.

Fraisse, Geneviève, [1989] 1995, Muse de la raison, démocratie et exclusion des femmes en France, Paris, Gallimard.

Gautier, Théophile, [1835] 1999, Mademoiselle de Maupin, Paris, Flammarion.

Gusdorf, George, 1982, Les Sciences humaines et la Pensée occidentale : fondements du savoir romantique, t. IX, Paris, Payot.

-, 1983, Les Sciences humaines et la Pensée occidentale : du néant à Dieu dans le savoir romantique, t. X, Paris, Payot.

Gutermann-Jacquet, Déborah, 2012, Les Équivoques du genre : devenir homme et femme à l'âge romantique, Rennes, Presses universitaires de Rennes.

Hugo, Victor, [1856] 2008, Les Contemplations, Paris, Flammarion.

Katz, Jonathan Ned, 2001, L'Invention de l'hétérosexualité, Paris, EPEL.

Laqueur, Thomas, 1990, Making Sex: Body and Gender from the Greeks to Freud, Cambridge, Mass, Havard University Press.

Maira, Daniel et Roulin, Jean-Marie (dir.), 2013, Masculinités en révolution de Rousseau à Balzac, Saint-Étienne, Publications de l'université de Saint-Étienne.

Minski, Alexandre, 2000, « La niche vide du Panthéon : monuments et beaux-arts de Rome », dans Michel Delon et Françoise Mélonio (dir.), Mme de Staël: Actes du colloque de la Sorbonne du 20 novembre 1999, Paris, Presses de l'Université Paris-Sorbonne, p. 17-30. 
Musset, Alfred de, [1836] 1973, La Confession d'un enfant du siècle, Paris, Gallimard.

Proudhon, Pierre-Joseph, [1912] 1989, Les femmelins : les grandes figures romantiques, Paris, À l'écart.

Rousseau, Jean-Jacques, [1762] 1834, Du contrat social, Paris, Lebigre.

Sand, George, [1833] 2003, Lélia, Paris, Gallimard.

-, [1846] 1894, Isidora, Paris, Calmann Lévy.

Schor, Naomi, 1993, George Sand and Idealism, New York, Columbia University Press.

Senancour, [1804] 1984, Obermann, Paris, Gallimard.

Staël Germaine de, [1802] 2000, Delphine, vol. 1 et 2, Paris, Gallimard.

-, [1807] 2006, Corinne ou l'Italie, Paris, Gallimard.

-, [1788] 2008, Lettres sur Rousseau, De l'influence des passions et autres essais moraux, Paris, Champion.

Stendhal, [1822] 1959, De l'amour, Paris, Garnier.

Tin, Luis-Georges, 2008, L'Invention de la culture hétérosexuelle, Paris, Autrement.

Vaillant Alain (dir.), 2012, Dictionnaire du romantisme, Paris, CNRS Éditions.

Wittig, Monique, [1992] 2007, La Pensée straight, Paris, Amsterdam.

Weber, Eugène, 1986, Fin de siècle, Paris, Fayard.

\section{NOTES}

1. Les ouvrages de Geneviève Fraisse, notamment, Muse de la raison, démocratie et exclusion des femmes en France ([1989] 1995), rendent compte de la modernisation de la rhétorique de la différence des sexes à partir du siècle des Lumières et ses conséquences au XIX ${ }^{\mathrm{e}}$ siècle.

2. La défaite de la France face à la Prusse en 1871 va dramatiser le besoin de viriliser une «France dégénérée ». Voir Eugen Weber, Fin de siècle (1986).

3. Poème «Réponse à un acte d'accusation » [1834], Les Contemplations, 1856.

4. Dans le Contrat Social, Rousseau conçoit la famille comme une miniaturisation de l'État: «La famille est donc si l'on veut le premier modèle des sociétés politiques : le chef est l'image du père, le peuple est l'image des enfants, et tous, étant nés égaux et libres, n'aliènent leur liberté que pour leur utilité » ([1762] 1834 : 23). On remarquera l'absence significative de la femme dans cette peinture politique de la famille.

5. Une autre question serait de savoir si le trouble hétérosexuel se décline également dans d'autres romantismes européens, et si oui, de quelle façon?

6. Voir l'article d'Alexandre Minski (2000).

7. Nous empruntons ce néologisme à Colette Guillaumin et Michèle Causse (2000).

8. Proudhon dans son pamphlet Les Femmelins: les grandes figures romantiques ([1912] 1989), accusera indistinctement des écrivains romantiques des deux sexes (Rousseau, Lamartine, Staël, Sand) d'être responsables de la « dévirilisation » de la France.

9. Lien de l'émission (Radio de la Communauté Juive du dimanche 2 juin 2013 ): https:// www.youtube.com/watch?v=qEBc6P9BvvE. 


\section{RÉSUMÉS}

La littérature romantique française peut être conçue comme un discours alternatif sur l'amour, la sexualité et l'identité de genre par opposition à la modernisation d'une norme sexuelle qui s'appuie sur la conjugaison des lois de la nature et des lois du Code civil pour assigner à chaque être humain un destin selon son sexe de naissance. Le concept de trouble hétérosexuel, fondé sur l'étude des couples hétérosexuels amoureux dans la littérature romantique française, démontre que la réinvention de l'amour vise à l'expérimentation d'une autre dialectique du féminin et du masculin pour proposer un nouveau contrat social aux sujets amoureux.

French Romantic literature can be understood as an alternative discourse on love, gender and sexuality in its opposition to the modernization of a normative sexuality grounded on the combination of the laws of nature and the laws of the Code civil in order to assign every human being a destiny stamped on the sex of his birth. The concept of heterosexual trouble, based on the study of heterosexual couples in love in French Romantic literature, illustrates that the reinvention of love aims at designing another dialectics of femininity and masculinity in order to articulate a new social contract for the subjects in love.

INDEX

Mots-clés : amour, romantisme, hétérosexualité, désordre, queer

Keywords : love, romanticism, heterosexuality, disorder, queer

\section{AUTEUR}

\section{MAXIME FOERSTER}

Southern Methodist University of Dallas 